TRANSACTIONS OF THE

AMERICAN MATHEMATICAL SOCIETY

Volume 180, June 1973

\title{
HIGHER DERIVATIONS AND FIELD EXTENSIONS
}

\author{
BY
}

\author{
R. L. DAVIS
}

ABSTRACT. Let $K$ be a field having prime characteristic $p$. The following conditions on a subfield $k$ of $K$ are equivalent: (i) $\bigcap_{n} K^{p^{n}}(k)=k$ and $K / k$ is separable. (ii) $k$ is the field of constants of an infinite higher derivation defined in $K$. (iii) $k$ is the field of constants of a set of infinite higher derivations defined in $K$. If $K / k$ is separably generated and $k$ is algebraically closed in $K$, then $k$ is the field of constants of an infinite higher derivation in $K$. If $K / k$ is finitely generated then $k$ is the field of constants of an infinite higher derivation in $K$ if and only if $K / k$ is regular.

Introduction. The relationship between field extensions and derivations was investigated by Baer [1] in 1927. Baer obtained a characterization of those subfields $k$ of the field $K$ that are the fields of constants of derivations defined in $K$. In the prime characteristic case it was found that $k$ is the field of constants of a nonzero derivation defined in $K$ if and only if $K / k$ is a purely inseparable extension having exponent one. Later Weisfield [7] generalized this result to finite higher derivations and purely inseparable extensions having higher exponent. The works of Weisfield [7] and Sweedler [6] yield the following: Let $K$ be a field having prime characteristic. The following conditions on a subfield $k$ of $K$ are equivalent:

(i) $K / k$ is a purely inseparable modular extension with finite exponent.

(ii) $k$ is the field of constants of a finite higher derivation in $K$.

(iii) $k$ is the field of constants of a set of finite higher derivations in $K$.

The purpose of this paper is to extend the above results to infinite higher derivations. The following is obtained: Let $K$ be a field having prime characteristic $p$. The following conditions on a subfield $k$ of $K$ are equivalent:

(i) $K / k$ is separable and $\bigcap_{n} K^{p^{n}}(k)=k$.

(ii) $k$ is the field of constants of an infinite higher derivation in $K$.

(iii) $k$ is the field of constants of a set of infinite higher derivations in $K$.

$A$ few comments should be made concerning the theory for characteristic zero fields. Baer [1] showed that in this case the subfields of $K$ which are fields of constants of derivations in $K$ are precisely those subfields algebraically closed in $K$. These subfields are also the fields of constants of the finite and infinite higher derivations in $K$.

Received by the editors February 22, 1971.

AMS (MOS) subject classifications (1970). Primary 12F 10, $12 \mathrm{~F} 15$.

Key words and phrases. Higher derivation, separable extension, separably generated, regular extension, $p$-basis.

Copyright $\odot 1973$, American Mathematical Society 
Preliminaries. All fields considered have prime characteristic $p$. Separable will mean separable in the linear disjoint sense. Let $K$ be a field.

Definition. An infinite higher derivation in $K$ is a sequence of additive mappings $\left(d_{i}\right)$ of $K$ into itself such that, for all $x$ and $y$ in $K$ and $n=0,1,2, \ldots$

$$
d_{n}(x y)=\sum\left\{d_{i}(x) d_{j}(y) \mid i+j=n\right\}
$$

and $d_{0}$ is the identity mapping of $K$.

The field of constants of a derivation is its kernel and the field of constants of a higher derivation $\left(d_{i}\right)$ is the intersection of the kernels of the $d_{i}$ for $i \geq 1$. If $k$ is a subfield of the field of constants of the derivation $d$ in $K, d$ is said to be a derivation in $K$ over $k$. The notation $\operatorname{Der}(K / k)$ is adopted for the collection of derivations in $K$ over $k$ and $H(K / k)$ is adopted for the analogous collection of infinite higher derivations in $K$.

The following results will be needed repeatedly in this paper.

Theorem A [3, p. 181]. Let $S$ be a p-basis for the field extension $K / k$ and $f: S \rightarrow K$ an arbitrary function. Then there exists a unique derivation $d$ in $K$ over $k$ such that $d(s)=f(s)$ for each $s \in A$.

Theorem B [2, Theorem 1]. Let $S$ be a p-basis for the separable field extension $K / k$ and $f:\{1,2, \cdots\} \times S \rightarrow K$ an arbitrary function. Then there exists a unique bigher derivation $\left(d_{i}\right)$ in $K$ over $k$ such that, for each $s \in S$ and $i \epsilon$ $\{1,2, \ldots\}, d_{i}(s)=f(i, s)$.

Theorem C [7, p. 436]. Let $\left(d_{i}\right)$ be a bigher derivation in $K$. Then for each $a \in K:(\mathrm{i}) d_{p j}\left(a^{p}\right)=\left(d_{j}(a)\right)^{p}$ for each $j \geq 1$ and (ii) $d_{j}\left(a^{p}\right)=0$ if $p$ does not divide $j$.

\section{Main result.}

Lemma 1. Let $K$ be a purely trancendental extension of $k$. Then there exists $d \in \operatorname{Der}(K)$ baving $k\left(K^{p}\right)$ as field of constants and $\left(d_{i}\right) \in H(K)$ with field of constants $k$.

Proof. Let $S$ be a transcendency basis for $K / k$ with $K=k(S)$. If $S=\left\{s_{1}\right.$, $\left.\cdots, s_{n}\right\}$ is finite, let $s_{0}=1$ and define $d\left(s_{i}\right)=\left(s_{0} \cdots s_{i-1}\right)^{-1}$ for $1 \leq i \leq n$. Since $S$ is a $p$-basis for $K / k^{\circ}$, this defines a unique derivation $d$ in $K$ over $k$ [Theorem A]. Let $0<m<n$ and $e^{(m)}$ denote the restriction of $d$ to $k\left(K^{p}\right)\left(s_{0}, \cdots, s_{m}\right)$. We induct on $m$ to show that the field of constants of $e^{(m)}$ is $k\left(K^{p}\right)$ and $\left(s_{0} \cdots s_{m}\right)^{-1} \notin \operatorname{Im}\left(e^{(m)}\right)$. If $m=0$, then the result is clearly true since $e^{(0)}$ is the zero derivation in $k\left(K^{p}\right)$. Thus assume the result for $m$ with $0<m<n$. 
Suppose there are $A_{i} \in k\left(K^{p}\right)\left(s_{0}, \cdots, s_{m}\right)$ such that

(1)

$$
e^{(m+1)}\left(\sum_{0}^{p-1} A_{i} s_{m+1}^{i}\right)
$$

$$
=\sum_{0}^{p-1} e^{(m)}\left(A_{i}^{\wedge}\right) s_{m+1}+\sum_{1}^{p-1} i A_{i} s_{m+1}^{i-1}\left(s_{0} \cdots s_{m}\right)^{-1}=0 .
$$

If $A_{i} \neq 0$ for some $i \geq 1$, then there exists a $j \geq 0$ such that $A_{j} \neq 0$ and $e^{(m)}\left(A_{j}\right)=0$. From (1) we have

$$
e^{(m)}\left(-A_{j-1} / j A_{j}\right)=\left(s_{0} \cdots s_{m}\right)^{-1} .
$$

This contradicts the induction hypothesis. Thus $A_{i}=0$ for each $i \geq 1$ and $A_{0} \epsilon$ $k\left(K^{p}\right)$ follows from the induction hypothesis.

Now suppose there are $B_{i} \in k\left(K^{p}\right)\left(s_{0}, \cdots, s_{m}\right)$ such that

$$
e^{(m+1)}\left(\sum_{0}^{p-1} B_{i} s_{m+1}^{i}\right)=\sum_{0}^{p-2}\left(e^{(m)}\left(B_{i}\right)+B_{i+1}(i+1)\left(s_{0} \cdots s_{m}\right)^{-1}\right) s_{m+1}^{i}
$$

or

$$
\begin{aligned}
& +e^{(m)}\left(B_{p-1}\right) s_{m+1}^{p-1} \\
= & \left(s_{0} \cdots s_{m+1}\right)^{-1}
\end{aligned}
$$

$$
\sum_{0}^{p-2}\left(e^{(m)}\left(B_{i}\right)+B_{i+1}(i+1)\left(s_{0} \cdots s_{m}\right)^{-1}\right) s_{m+1}^{i+1}+e^{(m+1)}\left(B_{p-1} s_{m+1}^{p}\right)
$$

$$
=\left(s_{0} \cdots s_{m}\right)^{-1} \text {. }
$$

Consequently, $e^{(m)}\left(B_{p-1} s_{m+1}^{p}\right)=\left(s_{0} \cdots s_{m}\right)^{-1}$ and this is a contradiction of the induction hypothesis.

If $S=\left\{s_{a}\right\}$ is infinite, well-order it so that there is no last element. Define $d\left(s_{a}\right)=s_{a+1}$ for each $s_{a} \in S$. This defines a unique derivation in $K$ over $k$ [Theorem A].

Let $A_{i} \in k\left(K^{p}\right)\left(\left\{s \in S \mid s<s_{a}\right\}\right)$ and suppose

$$
d\left(\sum_{0}^{p-1} A_{i} s_{a}^{i}\right)=\sum_{0}^{p-1} d\left(A_{i}\right) s_{a}^{i}+\sum_{1}^{p-1} i A_{i} s_{a}^{i-1} s_{a_{+1}}=0 .
$$

Necessarily, $\Sigma_{1}^{p-1} i A_{i} s_{a}^{i-1}=0$ and from this it follows that $A_{1}=\cdots=A_{p-1}=0$. Iteration of the process yields that $A_{0} \in k\left(K^{p}\right)$. Thus the field of constants of $d$ is $k\left(K^{p}\right)$.

Since the action of a higher derivation is completely determined by its action 
on a $p$-basis [Theorem B], there exists a higher derivation $\left(d_{i}\right) \in H(K / k)$ with $d_{1}=d$. Theorem $C$ is used to show that for each $i \geq 1$, the restriction of $d_{p^{i}}$ to $K^{p^{i}}(k)$ is a derivation. Since $d_{p^{i}}\left(x^{p^{i}}\right)=d(x)^{p^{i}}$ for each $x \in K$, we see that $d_{p^{i}}$ operates on $S^{p^{i}}$ in exactly the same manner $d$ operates on $S$. Since $S^{p^{i}}$ is a transcendency basis for $K^{p^{i}}(k) / k$ with $K^{p^{i}}(k)=k\left(S^{p^{i}}\right)$, the field of constants of the restriction of $d_{p^{i}}$ to $K^{p^{i}}(k)$ is $K^{p^{i+1}}(k)$. Thus the field of constants of $\left(d_{i}\right)$ is $\bigcap_{n} K^{p^{n}}(k)=\bigcap_{n}^{p^{i}} k\left(S^{p^{n}}\right)=k$.

Theorem 1. The following conditions on a subfield $k$ of $K$ are equivalent.

(i) $K / k$ is separable and $\bigcap_{n} K^{p^{n}}(k)=k$.

(ii) $k$ is the field of constants of a bigher derivation in $K$.

(iii) $k$ is the field of constants of a set of bigher derivations in $K$.

Proof. (i) implies (ii). Let $S$ be a $p$-basis for $K / k$ and $T$ be a $p$-basis for $k$. Since $S \cup T$ is a $p$-basis for $K$, it is algebraically independent over $K_{0}$, the maximal perfect subfield of $K$. The existence of an $\left(e_{i}\right) \in H\left(K_{0}(S \cup T)\right)$ with field of constants $K_{0}(T)$ is guaranteed by Lemma 1. In the proof of Lemma 1 it was shown that $\left(e_{i}\right)$ can be chosen such that the field of constants of the restriction of $e_{p j}$ to $K_{0}\left(S^{p j} \cup T\right)$ is $K_{0}\left(S^{p j+1} \cup T\right)$ for each $j \geq 0$. Take $\left(d_{i}\right)$ to be the unique higher derivation in $K$ agreeing with $\left(e_{i}\right)$ on $S \cup T$. Since $\left(d_{i}\right)$ acts trivially on $T, k$ is a subfield of the field of constants of $\left(d_{i}\right)$. Let $U$ be a linear basis for $K / K_{0}(S \cup T)$. We note that $U^{p}$ and hence $U^{p^{n}}$ for any $n$ is a linear basis for $K / K_{0}(S \cup T)$. It is easily verified that $K^{p}$ and $K_{0}(S \cup T)$ are linearly disjoint over $K_{0}\left(S^{p} \cup T^{p}\right)$. Thus $U^{p}$ is linearly independent over $K_{0}(S)$ and is a linear basis for $K^{p}(S \cup T)=K$ over $K_{0}(S \cup T)$.

Let $x \in K$ and assume $d_{i}(x)=0$ for all $i \geq 1$. We show that $x \in K^{p^{n}}(k)$ for each $n$ and consequently $x \in k$. Fix $\cdot n \geq 1$ and let $\left\{A_{i}\right\} \subseteq U$ and $\left\{a_{i}\right\} \subseteq K_{0}(S \cup T)$ be such that $x=\Sigma a_{i} A_{i}^{p^{n}}$. For each $1 \leq j<n$ we have

$$
d_{p^{j}}(x)=\sum d_{p^{j}}\left(a_{i}\right) A_{i}^{p^{n}}=\sum e_{p^{j}}\left(a_{i}\right) A_{i}^{p^{n}}=0 .
$$

As a consequence of the fact that each $e_{p_{j}}\left(a_{i}\right)=0$ we have that each $a_{i} \epsilon$ $K_{0}\left(S^{p^{n}} \cup T\right)$ and hence $x \in K^{p^{n}}(k)$. It now follows that the field of constants of $\left(d_{i}\right)$ is $k$.

(ii) implies (iii) is clear.

(iii) implies (i). Let $H$ denote a set of higher derivations and suppose $k$ is the field of constants of $H$. We first show that $\bigcap_{n} K^{p^{n}}(k)=k$. Let $x \in K^{p^{n}}(k)$ and $\left(d_{i}\right) \in H$. Fix $j \geq 1$ and let $p^{g}>j$. Since $x \in K^{p g}(k)$, there are $A$ and $B$ in $K^{p 8}[k]$ such that $x B=A$ and $B \neq 0$. We apply $d_{j}$ to both sides and use Theorem C to obtain that $d_{j}(x)=0$.

Now we need to show that $K^{p}$ and $k$ are linearly disjoint over $k^{p}$. If this 
were not the case, then there would exist a minimal subset of $k$ which is linearly independent over $k^{p}$ and linearly dependent over $K^{p}$. Thus suppose $\left\{A_{i}\right\}$ is such a minimal subset. Then

$$
\sum_{1}^{n} a_{i}^{p} A_{i}=0, \quad\left\{a_{i}\right\} \subseteq K, \quad \text { each } a_{i} \neq 0, n>1 .
$$

Without loss of generality we may assume $a_{1}=1$ and $a_{2} \notin k$. There exists a $\left(d_{i}\right) \in H$ and $j>0$ such that $d_{j}\left(a_{2}\right) \neq 0$.

$$
d_{p j}\left(A_{1}+\sum_{2}^{n} a_{i}^{p} A_{i}\right)=\sum_{2}^{n}\left(d_{j}\left(a_{i}\right)\right)^{p} A_{i}=0 .
$$

The minimal nature of $n$ is contradicted by (8). Thus $K / k$ is a separable field extension.

Theorem 2. If $K / k$ is separably generated and $k$ is algebraically closed in $K$, then there exists an infinite bigher derivation in $K$ baving field of constants $k$.

Proof. Let $S$ be a separating transcendency basis for $K / k$. An application of Theorem 1 to the field extension $k(S) / k$ yields the existence of a higher derivation $\left(d_{i}\right)$ defined on $k(S)$ having $k$ as field of constants. Since $S$ is a $p$-basis for $K$, there is a unique extension of $\left(d_{i}\right)$ to $K / k$ [Theorem B]. We now show that the field of constants of this extension is $k$. Suppose $s$ is an element of the field of constants of $\left(d_{i}\right)$. Let $f(x)=x^{m}+f_{m-1} x^{m-1}+\cdots+f_{0}$ denote the minimal polynomial of $s$ over $k(S)$. If each $f_{i} \in k$, then $s$ is algebraic over $k$ and we are finished. So suppose some $f_{i} \notin k$. Thus there exists $q>0$ such that $d_{q}\left(f_{i}\right) \neq 0$. We may assume $q$ to be minimal and that $d_{j}\left(f_{\boldsymbol{g}}\right)=0$ for each $g$ and each $j<q$.

$$
d_{q}(f(s))=d_{q}\left(s^{m}+\sum_{0}^{m-1} f_{i} s^{i}\right)=\sum_{0}^{m-1} d_{q}\left(f_{i}\right) s^{i}=0 .
$$

The polynomial $\Sigma_{0}^{m-1} d_{q}\left(f_{i}\right) x^{i}$ is nonzero and has degree less than that of the minimal polynomial of $s$. This is a contradiction. Thus each $f_{i} \in k$ and hence $s \in k$.

Corollary 1. If $K / k$ is separably generated and $k$ is algebraically closed in $K$, then $\bigcap_{n} K^{p^{n}}(k)=k$.

Recall that a field extension $K / k$ is said to be regular if $K$ and $\bar{k}$ are linearly disjoint over $k$. Regularity is equivalent to $K / k$ being a separable extension and $k$ being algebraically closed in $K[4$, p. 56]. Thus we have

Corollary 2. Let $K / k$ be finitely generated. Then $k$ is the field of constants of a bigher derivation in $K$ if and only if the extension $K / k$ is regular.

The following example due to Mac Lane [5] illustrates that the converse of 
Theorem 2 is false. Let $k$ be a perfect field and let $T=\left\{t_{0}, t_{1}, \ldots\right\}$ be algebraically independent over $k$, define $Y=\left\{y_{2}, y_{3}, \ldots\right\}$ by $y_{n}^{p}=t_{n-2}+t_{n-1} t_{n}^{p}, n \geq 2$, and let $K=k(T)(Y)$. Then $K / k$ is a separable extension having $\bigcap_{n} K^{p^{n}}(k)=k$; however, $K / k$ is not separably generated.

\section{REFERENCES}

1. R. Baer, Algebraische Theorie der differentierbaren Runktionenkorper. I, S.-B. Heidelberg. Akad. 1927, 15-32.

2. N. Heerema, Derivations and embeddings of a field in its power series ring. II, Michigan Math. J. 8 (1961), 129-134. MR $25 \# 69$.

3. N. Jacobson, Lectures in abstract algebra. Vol. 3: Theory of fields and Galois theory, Van Nostrand, Princeton, N. J., 1964. MR 30 \#3087.

4. S. Lang, Introduction to algebraic geometry, Interscience, New York, 1958. MR $20 \# 7021$.

5. S. Mac Lane, Steinitz field towers for modular fields, Trans. Amer. Math. Soc. 46 (1939), 23-45. MR 1, 3.

6. M. Sweedler, Structure of inseparable extensions, Ann. of Math. (2) 87 (1968), 401-410. MR $36 \# 6391$.

7. M. Weisfeld, Purely inseparable extensions and higher derivations, Trans. Amer. Math. Soc. 116 (1965), 435-449. MR $33 \# 122$.

DEPARTMENT OF MATHEMATICS, LOUISIANA STATE UNIVERSITY, BATON ROUGE, LOUISIANA 70803 